\title{
MHD Pressure Driven Two Phase flow in an inclined channel contain Porous medium
}

\author{
Y. Shagaiya Daniel and Simon Daniel \\ Department of Mathematics Kaduna State University, Kaduna, Nigeria
}

\begin{abstract}
The problem of two-phase MHD flow in an inclined channel contain porous medium has been considered. The differential equations governing the flow and heat transfer are solved analytically in both fluid regions of the channel. Effects of physical parameter such as Pressure parameter, ratio of heights, Hartmann number and ratio of viscosities on the velocity and temperature fields are shown graphically. It was found that the pressure parameter on both the velocity and temperature drop as result of magnetic field and porous material. Results have been presented for a wide range of governing parameters.
\end{abstract}

Keywords: incline channel, porous medium, electromagnetic force,

\section{Nomenclature}

$c_{p}$ specific heat at constant pressure

gacceleration due to gravity

$h$ ratio of the heights of the region $\left(\frac{h_{2}}{h_{1}}\right)$

$h_{1}$ height of the region $-\mathrm{I}$

$h_{2}$ height of the region - II

Re Reynolds number $\left(\frac{E_{0}}{B_{0} u_{0}}\right)$

$k$ permeability of the porous medium

Kratio of the thermal conductivities $\left(\frac{k_{1}}{k_{2}}\right)$

$k_{1}$ effective thermal conductivity of the fluid saturated porous medium in region- I

$k_{2}$ thermal conductivity of the fluid in region-II

mratio of the viscosities $\left(\frac{\mu_{1}}{\mu_{2}}\right)$

ppressure

Pnondimensional pressure gradient $\left(-\frac{h_{1}^{2}}{\mu_{1} u_{0}} \frac{d p^{\prime}}{d x^{\prime}}\right)$

$\operatorname{Pr}$ Prandtl number $\left(\frac{\mu_{1} c_{p}}{k_{1}}\right)$

Ttemperature

$T_{w 1}, T_{w 2}$ temperature of the boundaries

uvelocity

M Hartmann number $\left(\frac{\sigma_{1} B_{0}^{2} h_{1}^{2}}{\rho_{1} \vartheta_{1}}\right)$

$x, y, z$ space coordinates

\section{Greek Symbols}

$\beta$ ratio of the coefficients of the thermal expansion $\left(\frac{\beta_{2}}{\beta_{1}}\right)$

$\sigma$ porous parameter $\left(\frac{h_{1}}{\sqrt{k}}\right)$

$\rho$ density of the fluid

$\vartheta$ kinematic viscosity

$\mu$ viscosity

$\theta$ nondimensional temperature $\left(\frac{T-T_{w}}{\frac{u_{0}^{2} \vartheta_{1}}{k_{1}}}\right)$

Subscripts

$1 \& 2$ refers to the quantities for region I and II respectively

$w$ wall conditions 


\section{Introduction}

The importance and application of fluid flows and heat transfer in petroleum transport, wastewater treatment, combustion, power plant piping, corrosive particles in engine oil flows, etc. Particularly, the flow and heat transfer of electrically conducting fluids in an inclined channel contain porous medium under the effect of a transverse magnetic field occurs in magnetohydrodynamic (MHD) generators,

Pumps, accelerators, and flow meters and has possible applications in nuclear reactors, filtration, geothermal systems and others. As a result of the growing interest in the latter, a more accurate understanding of heat transfer phenomena containing porous continues to attract attention cheng [1] and Al-Nimr and Haddad [2] studied the convective flow and heat transfer in a vertical channel partially filled with porous media. Jain and Mehta [3] examined wall suction/injection effects on the Globe problem. Bathaiah and Venupogal [4] examined the MHD flow between two concentric rotating cylinders under the influence of a uniform magnetic field, with non-erodable and non-conducting porous lining on the inner wall of the outer cylinder under the influence of a uniform radial magnetic field of the form $B_{r}=A / r$ where $r$ is radial coordinate, discussing the effects of ratio of the velocities of the cylinder, the slip parameter, the porosity parameter and the Biot number on the flow variables Prasad et al [5] studied numerically the influence of Hall currents and Oblique applied magnetic field on rotating plasma MHD flow in a two-dimensional channel at generalized Eckman numbers. Recently Beg et al [6] obtained comprehensive network computational solutions for the two-dimensional transient hydromagnetic flow in a parallel-plate channel containing a Darcian porous material with dissipation and Hall/ion slip currents. The boundary layer and inertial effects on vertical surface in porous medium have been examined by Hseieh et al [7], and chien et al [8]. Jet et al [9] studied hydromagnetic flow and heat transfer on a continuously moving surface. He has studied the effect of parameters such as reference temperature, exponent, magnetic, Prandtl number and Eckert number on heat transfer.

Therefore, the objective of this study is to present a unified approach to the flow and heat transfer in an inclined channel contain porous medium saturated with a fluid and a clear viscous fluid layer. The fluid is subjected to a uniform suction from above and a uniform injection from below and mass conservation is applied perpendicular to the plates while no electric field is applied and the induced magnetic field is neglected by assuming a very small magnetic Reynolds number. The effect of the Pressure parameter, Porous parameter, Ratio of heights of the two layers, Ratio of the viscosities and permeability of the porous medium on the velocity and temperature fields are reported.

\section{Mathematical Analysis}

We considered infinite inclined parallel plates maintained at different constant temperatures, extending in the $z$ and $x$-directions. Mathematically the problem involves the coupling of the governing equations for the fluid region with the equations for the porous region through an appropriate set of matching conditions at the fluid/porous medium interface. We assume the continuity of velocity, shear stress, temperature and heat flux at the interface. The region $o \leq y \leq h_{1}$ is filled with porous matrix and the region $-h_{2} \leq y \leq 0$ is occupied by clear fluid. The two walls of the channel are held at different temperature $\Delta T=T_{w 1}-T_{w 2}$ where $T_{w 1}$ is the temperature of the boundary at $y=h$ and $T_{w 2}$ is the temperature at the boundary at $y=-h$. In the analysis, the porous medium is considered to be homogeneous. The fluid within the porous medium saturates the solid matrix and both are in local thermodynamic equilibrium. The flow is assumed to be steady, laminar and incompressible. The fluid in both regions is assumed to be deriven by a common constant pressure gradient $-\frac{\partial p}{\partial x}$ and that existence of heat transfer does not affect the pressure gradient.

Region I (Porous region)

$$
\begin{aligned}
V_{0} \frac{d u_{1}^{\prime}}{d y^{\prime}} & =\vartheta_{1} \frac{d^{2} u_{1}}{d y^{\prime 2}}-\frac{\partial p^{\prime}}{\partial x^{\prime}} \frac{1}{\rho_{1}}-\frac{\sigma B_{0}^{2}}{\rho_{1}}\left[u_{1}^{\prime}+\frac{E_{0}}{B_{0}}\right] \\
V_{0} c_{p} \frac{d T_{1}}{d y^{\prime}} & =\frac{k_{1}}{\mu_{1}} \frac{d^{2} T_{1}^{\prime}}{d y^{\prime 2}}+\left(\frac{d u_{1}}{d y}\right)^{2}+\frac{M^{2} u_{1}^{2}}{k}
\end{aligned}
$$

Region II (clear fluid region)

$$
\begin{aligned}
V_{0} \frac{d u_{2}^{\prime}}{d y^{\prime}} & =\vartheta_{2} \frac{d^{2} u_{2}}{d y^{\prime 2}}-\frac{\partial p^{\prime}}{\partial x^{\prime}} \frac{1}{\rho_{2}} \\
V_{0} c_{p} \frac{d T_{2}}{d y^{\prime}} & =\frac{k_{2}}{\mu_{2}} \frac{d^{2} T_{2}^{\prime}}{d y^{\prime 2}}+\left(\frac{d u_{2}}{d y}\right)^{2}+\frac{u_{2}^{2}}{k}
\end{aligned}
$$

The appropriate boundary and matching conditions for the problem under consideration can be written as

$$
\begin{aligned}
& u_{1}\left(h_{1}\right)=0 \\
& u_{1}(0)=u_{2}(0) \\
& u_{2}\left(-h_{2}\right)=0
\end{aligned}
$$


$\mu_{1} \frac{d u_{1}}{d y}=\mu_{2} \frac{d u_{2}}{d y}$ aty $=0$

$$
T_{1}(0)=T_{2}(0)
$$

$$
\begin{gathered}
T_{1}\left(h_{1}\right)=T_{w 1} \\
T_{2}\left(-h_{2}\right)=T_{w 2}
\end{gathered}
$$

$k_{1} \frac{d T_{1}}{d y}=k_{2} \frac{d T_{2}}{d y}$ aty $=0$

In order to non-dimensionalise the governing equations the following transformations are used

$$
\begin{aligned}
& y=\frac{y^{\prime}}{h}, \quad u=\frac{u^{\prime}}{u_{0}}, \quad P=-\frac{h_{1}^{2}}{\vartheta_{1} u_{0}} \frac{d p^{\prime}}{d x^{\prime}}, \quad R e=\frac{E_{0}}{B_{0} u_{0}}, \quad s=\frac{V_{0} h_{1}}{\vartheta_{1}}, \quad \operatorname{Pr}=\frac{\mu_{1} c_{p}}{k_{1}}, \quad \gamma=\frac{h_{1}}{\sqrt{k}} \\
& M=\frac{\sigma_{1} B_{0}^{1} h_{1}^{2}}{\rho_{1} \vartheta_{1}}, \quad \theta=\left(\frac{T-T_{w}}{\frac{u_{0}^{2} \vartheta_{1}}{k_{1}}}\right), \quad \delta=\frac{\vartheta_{1}}{u_{0}}, \quad \beta=\frac{1}{\vartheta_{1}},
\end{aligned}
$$

The ratios of different parameters that appears in governing equations are

$$
h=\frac{h_{2}}{h_{1}}, \quad k=\frac{k_{1}}{k_{2}}, \quad m=\frac{\mu_{1}}{\mu_{2}}
$$

Substitution of the above nondimensional quantities in equations (1)-(4), results in the following equations.

Region-I

Region-II

$$
\begin{aligned}
& s \frac{d u_{1}}{d y}=\frac{d^{2} u_{1}}{d y^{2}}+P-M\left(u_{1}+R e\right) \\
& s \operatorname{Pr} \delta \frac{d \theta_{1}}{d y}=\frac{d^{2} \theta_{1}}{d y^{2}}+\left(\frac{d u_{1}}{d y}\right)^{2}+\gamma^{2} u_{1}^{2}
\end{aligned}
$$

$$
\begin{aligned}
& \operatorname{sh} \alpha \frac{d u_{2}}{d y}=\frac{d^{2} u_{2}}{d y^{2}}-+P \Gamma h^{2} \\
& \frac{\operatorname{shPr}}{\beta} \frac{d \theta_{2}}{d y}=\frac{d^{2} \theta_{2}}{d y^{2}}+\left(\frac{d u_{2}}{d y}\right)^{2}
\end{aligned}
$$

The transformed boundary and matching conditions are given by

$$
\frac{d u_{1}}{d y}=\frac{1}{m h} \frac{d u_{2}}{d y} \text { at } y=0
$$

$$
\begin{gathered}
u_{1}(1)=0 \\
u_{1}(0)=u_{2}(0) \\
u_{2}(-1)=0
\end{gathered}
$$

$$
d y \quad m h d y
$$

$\frac{d \theta_{1}}{d y}=\frac{1}{k h} \frac{d \theta_{2}}{d y}$ at $y=0$

$$
\begin{gathered}
\theta_{1}(1)=1 \\
\theta_{1}(0)=\theta_{2}(0) \\
\theta_{2}(-1)=0
\end{gathered}
$$

Solving the differential equations (8), (9), (10) and (11) satisfying the boundary and interface conditions (12) and (13) we obtain

$$
\begin{aligned}
& u_{1}(y)=\exp \left(\frac{s y}{2}\right)\left[c_{1} \cosh (\lambda y)+c_{2} \sinh (\lambda y)\right]-R e+ \\
& \frac{P}{M} \quad \text { (14)where } \lambda=\frac{\sqrt{s^{2}+4 M}}{2} \\
& \theta_{1}(y)=A_{1} \sinh (2 \lambda y)+A_{2} \cosh (2 \lambda y)+A_{3} \sinh (\lambda y)+A_{4} \cosh (\lambda y)+A_{5} \exp (\lambda y)+A_{6} y+A_{7} \\
& +c_{5} y \exp (s \operatorname{Pr} \delta y)+c_{6} \exp (s \operatorname{Pr} \delta y) \\
& u_{2}(y)=\left(P \Gamma h^{2}\right)\left[y+\frac{1}{s h \alpha}\right]+c_{4} \operatorname{sh} \alpha \exp (\operatorname{sh} \alpha y)-c_{3} \\
& \theta_{2}(y)=B_{1} y+B_{2}+B_{3} \exp (\operatorname{sh} \alpha y)+B_{4} \exp (2 \operatorname{sh} \alpha y)-c_{7} y \exp \left(\frac{\operatorname{shPry}}{\beta}\right)-c_{8} \exp \left(\frac{\operatorname{shPry}}{\beta}\right)
\end{aligned}
$$

The constants appearing in equations (14)-(17) are presented in the appendix. The solutions for the equations given by (14)-(17) are evaluated numerically, fixing some of the parameters and varying the parameters. 


\section{Results and Discussion}

In order to have a physical insight into the problem, we have written a MATLAB programme to compute and generate the graphs for the velocity and temperature of the two different phases. Some result are presented in the form of line graphs in fig. 1-8 to interpret the effects of these Parameters, For fixing some of the parameters namely $\Gamma=\lambda=\operatorname{Re}=\gamma=\beta=1, \alpha=10, s=5, \operatorname{Pr}=0.71, k=10$, and $\delta=2$ and varying the parameters $P, h, M$, and $m$.

The effect of pressure parameter $p$ on velocity and temperature field is shown in figs. 1 and 2. For fixed values of other parameters pressure drops in region-I as result of porous materials and magnetic field and increase in clear fluid region, which is insignificant cause is almost flat in region-II

The effect of varying the height ratio $h$ is shown in figs 3 and 4 . An increase in the value of $h$ increases both the velocity and temperature profiles. It is found that the larger the ratio of the height more the amount of heat transfer. For fixing $\beta=10$ and $\Gamma=0.1$ and other values remained constant

The effects of varying the Hartmann number $M$ on velocity and temperature profiles are shown in figs. 5 and 6 . We observed that the effect of electromagnetic force is to accelerate the flow. As Hartmann number $M$ increases both the velocity and temperature increases.

The effect of ratio of viscosities $m$, on velocity and temperature are shown in figs. 7 and 8 . As the ratio of viscosities of porous region increases, the fluid in clear region is almost flat as result of friction force. The clear fluid is more viscos than the porous region on velocity and temperature profiles. As velocity and temperature increases, ratio of the viscosities increases.

\section{Conclusions}

The MHD Pressure driven two phase flow in an inclined channel with porous matrix is considered. The governing differential equation is solved analytically. The effects of pressure parameter $p$, ratio of heights $h$, Hartmann number $M$,and ratio of viscosities $m$. It is observed that pressure parameter increases as velocity and temperature increases in the presence of magnetic field and porous matrix, which is fixed relative to the fluid. The magnetic field has a retarding influence on the flow field. Also ratio of the heights increases the amount of heat transfer. The ratio of viscosities and electromagnetic force accelerate the flow in the presence of porous matrix.

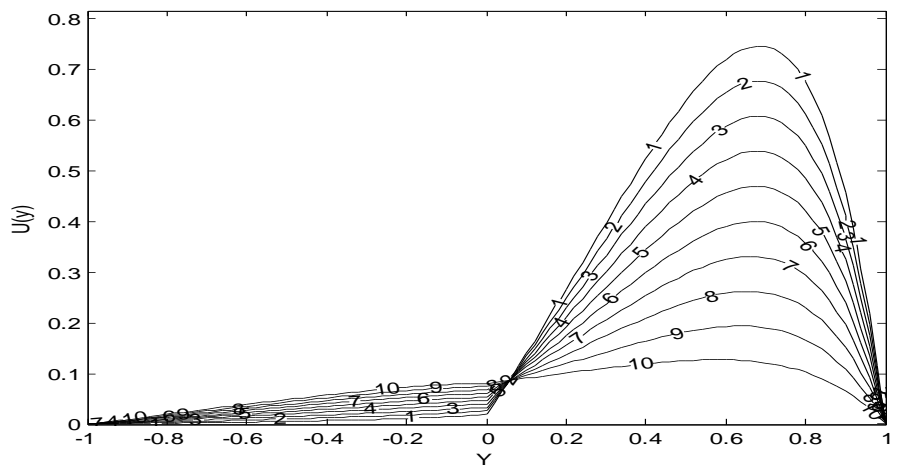

Fig.1 Velocity profiles showing effect of Pressure ParameterP

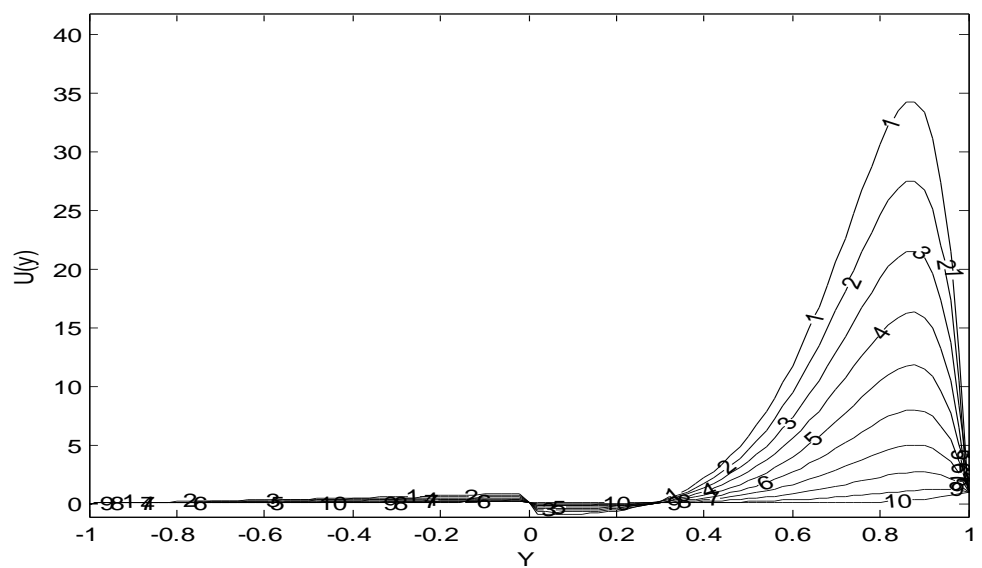

Fig.2 Temperature profiles showing effect of Pressure ParameterP 


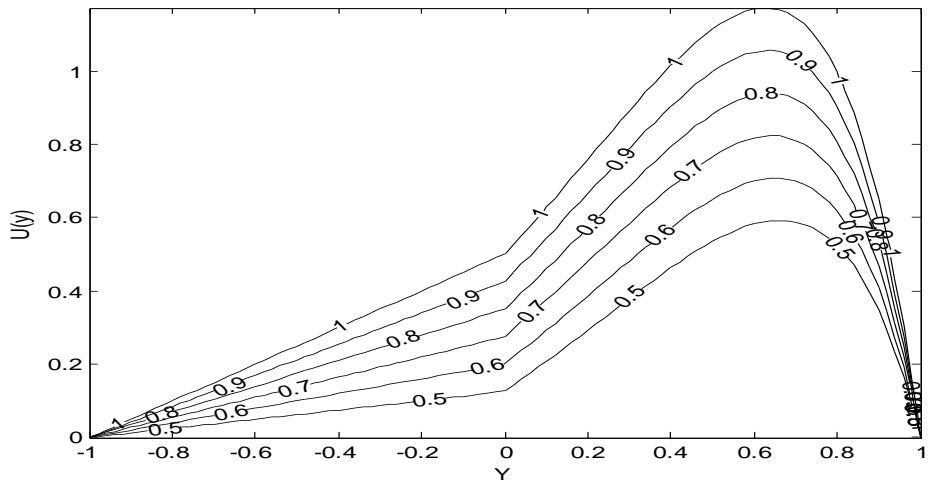

Fig.3Velocity profiles showing effect of ratio of heights $h$

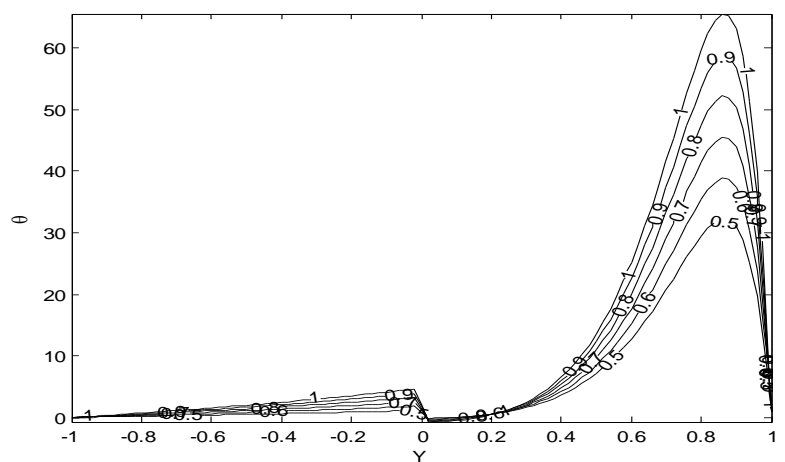

Fig.4Temperature profiles showing effect of Ratio of heights $h$

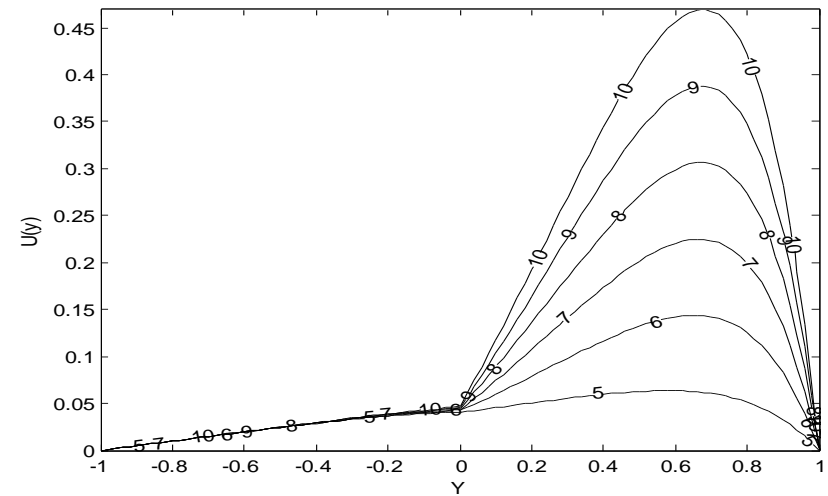

Fig.5Velocity profiles showing effect of Ratio of Hartmann number $M$

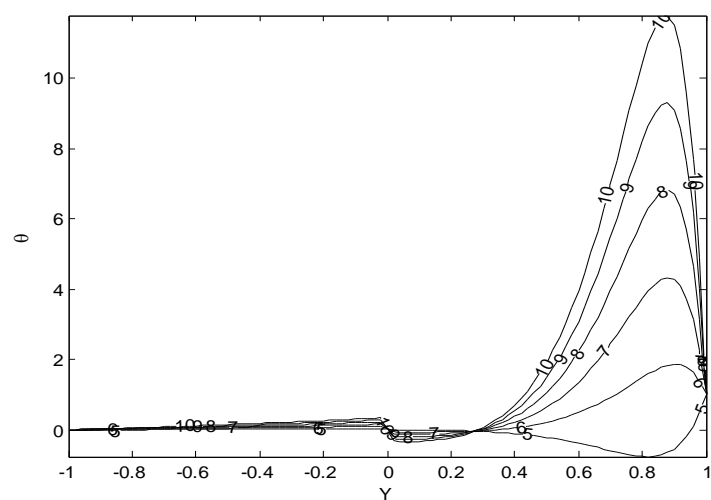

Fig.6Temperature profiles showing effect of Ratio of Hartmann number $M$ 


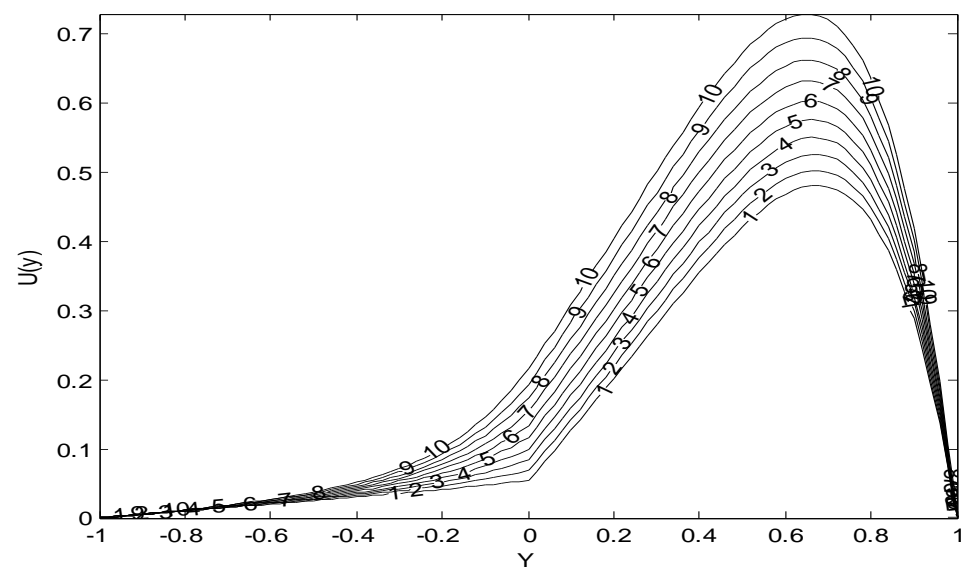

Fig.7Velocity profiles showing effect of Ratio of viscosities $m$

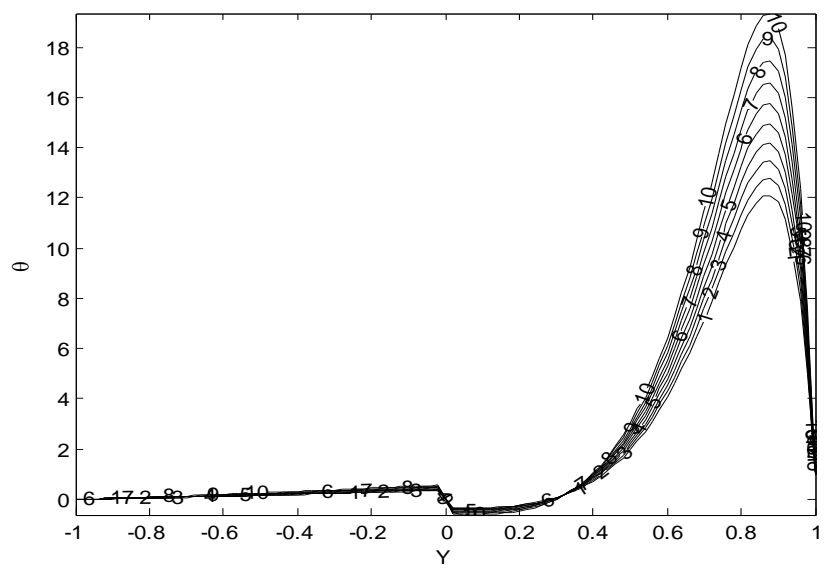

Fig.8Temperature profiles showing effect of Ratio of viscosities $m$

\section{Appendix}

$$
\begin{aligned}
& N_{1}=R e-\frac{P}{M}, \quad N_{2}=\cosh (\lambda) \exp \left(\frac{S}{2}\right), \quad N_{3}=\sinh (\lambda) \exp \left(\frac{S}{2}\right), \quad N_{4}=\left(P \Gamma h^{2}\right)\left[\frac{1}{\operatorname{sh} \alpha}-1\right] \\
& N_{5}=(\operatorname{sh} \alpha) \exp (-s h \alpha), \quad N_{6}=(m h)\left(\left(\frac{s}{2}\right)-\left(\frac{N_{2 \lambda}}{N_{3}}\right)\right), \quad N_{8}=(\operatorname{sh} \alpha)-N_{5}, \quad N_{9}=\frac{\left(P \Gamma h^{2}\right)}{\operatorname{sh} \alpha} \\
& N_{10}=N_{9}-N_{4}+N_{1}, \quad N_{11}=\frac{N_{1} m h}{N_{3}}+N_{10} N_{6}-P \Gamma h^{2}, \quad N_{12}=(\operatorname{sh} \alpha)^{2}-N_{8} N_{6}, \quad c_{4}=\frac{N_{11}}{N_{12}}, \\
& N_{13}=\frac{\left(c_{1}^{2}+c_{2}^{2}\right)}{2}\left[\lambda^{2}-\left(\frac{s}{2}\right)^{2}\right]+\left[\left(\frac{s}{2}\right) c_{1}^{2}+\lambda^{2} c_{2}^{2}\right], \quad N_{14}=\frac{\left(c_{1}^{2}+c_{2}^{2}\right)}{2}\left(\frac{s}{2}\right)^{2}, \\
& N_{15}=\frac{\left(c_{1}^{2}+c_{2}^{2}\right)}{2} \lambda^{2}, \quad N_{16}=c_{1} c_{2}\left[\left(\frac{s}{2}\right)^{2}+\lambda^{2}\right], \quad N_{17}=c_{1} c_{2} s \lambda, \quad N_{18}=N_{14}+N_{15}+N_{17} \\
& N_{19}=\frac{\left(c_{1}^{2}-c_{2}^{2}\right)}{2}, \quad N_{20}=\frac{\left(c_{1}^{2}+c_{2}^{2}\right)}{2}, \quad N_{21}=c_{1} c_{2}, \quad N_{22}=\frac{P}{M}-R e, \\
& N_{23}=\frac{N_{18}+(\gamma M)^{2} N_{20}}{2 \lambda-\frac{s^{2}}{2 \lambda}}, \quad N_{24}=\frac{N_{17}+(M \gamma)^{2} N_{21}}{2 \lambda-\frac{s^{2}}{2 \lambda}}, \quad N_{25}=\frac{N_{13}-(M \gamma)^{2} N_{19}}{s} \text {, } \\
& N_{26}=\frac{2 c_{1} N_{22}}{\lambda-\frac{s^{2}}{4 \lambda}} \\
& N_{27}=\frac{2 c_{2} N_{22}}{\lambda-\frac{s^{2}}{4 \lambda}}, \quad A_{1}=\frac{\left(\frac{s N_{23}}{2 \lambda}-N_{24}\right)-s\left(\frac{s N_{24}}{2 \lambda}-N_{23}\right)\left(\frac{1-P r \delta}{2 \lambda}\right)}{2 \lambda-\frac{s^{2}(1-\operatorname{Pr} \delta)^{2}}{2 \lambda}}
\end{aligned}
$$




$$
\begin{aligned}
& A_{2}=\frac{\left(\frac{s N_{24}}{2 \lambda}-N_{23}\right)-s\left(\frac{s N_{23}}{2 \lambda}-N_{24}\right)\left(\frac{1-P r \delta}{2 \lambda}\right)}{2 \lambda-\frac{s^{2}(1-\operatorname{Pr} \delta)^{2}}{2 \lambda}}, \quad A_{3}=\frac{\left(\frac{s N_{26}}{2 \lambda}-N_{27}\right)-s\left(\frac{s N_{27}}{2 \lambda}-N_{26}\right)\left(\frac{\frac{1}{2}-\operatorname{Pr} \delta}{2 \lambda}\right)}{2 \lambda-\frac{s^{2}\left(\frac{1}{2}-\operatorname{Pr} \delta\right)^{2}}{2 \lambda}} \\
& A_{4}=\frac{\left(\frac{s N_{27}}{2 \lambda}-N_{26}\right)-s\left(\frac{s N_{26}}{2 \lambda}-N_{27}\right)\left(\frac{\frac{1}{2}-P r \delta}{2 \lambda}\right)}{2 \lambda-\frac{s^{2}\left(\frac{1}{2}-P r \delta\right)^{2}}{2 \lambda}} \\
& B_{1}=\frac{\beta\left(P \Gamma h^{2}\right)^{2}}{s h P r}, \quad B_{2}=\left(\frac{\beta}{s P r h}\right)^{2}\left(P \Gamma h^{2}\right)^{2}, \quad B_{3}=-\frac{2 c_{4}\left(P \Gamma h^{2}\right) s h \alpha}{s h\left[\alpha-\frac{P r}{\beta}\right]}, \quad B_{4}=-\frac{c_{4}^{2}(s h m)^{3}}{2\left[\operatorname{sh}\left(\alpha-\frac{P r}{2 \beta}\right)\right]} \\
& N_{28}=A_{1} \sinh (2 \lambda)+A_{2} \cosh (2 \lambda)+A_{3} \sinh (\lambda)+A_{4} \cosh (\lambda)+A_{5} \exp (s)+A_{6}+A_{7} \\
& N_{29}=B_{2}-B_{1}+B_{3} \exp (-s p \alpha)+B_{4} \exp (-2 s h \alpha), \quad N_{30}=A_{2}+A_{4}+A_{5}+A_{7} \\
& N_{31}=B_{2}+B_{3}+B_{4}, \quad N_{32}=2 \lambda A_{2}+A_{4} \lambda+A_{5} s+A_{6}, \quad N_{33}=B_{1}+B_{3}(\operatorname{sh} \alpha)+2(\operatorname{shm}) B_{4} \\
& N_{34}=\left[N_{33}-\frac{N_{29}\left(\frac{s h P r}{\beta}\right)}{\exp \left(-\frac{s h P r}{\beta}\right)}\right]-\left[N_{32}(h k)+\frac{(s \operatorname{Pr} \delta h k)\left(1-N_{28}\right)}{\exp (s \operatorname{Pr} \delta)}\right], \quad N_{35}=(h k)[1-(s \operatorname{Pr} \delta)] \\
& N_{36}=\frac{s h P r}{\beta}-1, \quad N_{37}=1+\frac{N_{36}}{N_{35}}, \quad N_{38}=\left[N_{30}+\frac{1-N_{28}}{\exp (\operatorname{sPr} \delta)}\right]-\left[\frac{N_{34}}{N_{35}}+N_{31}+\frac{N_{29}}{\exp \left(-\frac{s h P r}{\beta}\right)}\right] \\
& c_{7}=\frac{N_{38}}{N_{37}}, \quad c_{5}=\frac{c_{7} N_{36}+N_{34}}{N_{35}}, \quad c_{6}=\frac{\left(1-N_{28}\right)-c_{5} \exp (\mathrm{s} \operatorname{Pr} \delta)}{\exp (\mathrm{s} \operatorname{Pr} \delta)}, \quad c_{8}=\frac{N_{29}+c_{7} \exp \left(-\frac{\operatorname{shPr}}{\beta}\right)}{\exp \left(-\frac{\operatorname{shPr}}{\beta}\right)}
\end{aligned}
$$

\section{Reference}

[1] Cheng W, Cheng W (1996) mixed convective in a vertical parallel-plate channel partially filled with porous media of high permeability. Int. J. Heat Mass Transfer 39:1331

[2] Al-Nimr MA, Haddad OM (1999) Fully developed free convection in open-ended vertical channels partially filled with porous materials J. Porous Media 2:179

[3] Jain R.K and Mahta KN (1962) Laminar hydromagnetic flow in an annulus with porous walls pysics of fluids 5(10) 1207-1211

[4] Bathaiah D. and Venugopal R. (1982) Effects of porous lining of the MHD flow between two concentric rotating cylinders under the influence of a uniform magnetic field, ActaMechanica, 44, 3-4 141-158

[5] Prasad VR, Takhar HS, Zucco J, Ghosh SK, and Beg OA (2008) Numerical study of hydromagnetic viscous plasma flow with Hall current effects in rotating porous media $53^{\text {rd }}$ congress Indian society Theoretical and Applied Mechanics, University college of Engineering, Osmania University, Hyderabad, India December, 147-157

[6] Beg OA Zucco J. and Takhar HS (2009) Unsteadymagnetohydrodynamic Hartmann-Coutte flow with Hall current, ionslip, Viscous and Joule heating effects: Network numerical solutions communications in Nonlinear science and Numerical Simulation, 14 10821097

[7] J.C Hsieh, TS Chen, BF Armoly, (1993)Mixed convection along a nonisothermal vertical flat plate embedded in a porous medium, intl. J. Heat Mass Transfer 36(7) 1819-1825

[8] C-H Chen, TS Chen, C-K, Chen, (1996) Non-Darcy mixed convection along nonisothermal vertical surface in porous media, Intl. J. Heat Mass Transfer 36(6), 1157-1164

[9] Jat, R.N, and SantoshChaudhary (2010) Hydromagnetic flow and Heat transfer on a continuously flow and Heat transfer on a continuously moving surface. Applied Mathematical Sciences, 4; 2; 65-78 